\title{
Fault tolerant control - a residual based set-up
}

\author{
Niemann, Hans Henrik; Poulsen, Niels Kjølstad
}

\section{Published in:}

Proceedings of the 48th IEEE Conference on Decision and Control, 2009 held jointly with the 2009 28th Chinese Control Conference. CDC/CCC 2009

Link to article, DOI:

10.1109/CDC.2009.5400876

Publication date:

2009

Document Version

Publisher's PDF, also known as Version of record

Link back to DTU Orbit

Citation (APA):

Niemann, H. H., \& Poulsen, N. K. (2009). Fault tolerant control - a residual based set-up. In Proceedings of the 48th IEEE Conference on Decision and Control, 2009 held jointly with the 2009 28th Chinese Control Conference. CDC/CCC 2009 (1 ed., pp. 8470-8475). IEEE. https://doi.org/10.1109/CDC.2009.5400876

\section{General rights}

Copyright and moral rights for the publications made accessible in the public portal are retained by the authors and/or other copyright owners and it is a condition of accessing publications that users recognise and abide by the legal requirements associated with these rights.

- Users may download and print one copy of any publication from the public portal for the purpose of private study or research.

- You may not further distribute the material or use it for any profit-making activity or commercial gain

- You may freely distribute the URL identifying the publication in the public portal 


\section{Fault Tolerant Control - A Residual based Set-up}

\author{
Henrik Niemann \\ Dept. of Elec. Engineering \\ Automation and Control \\ Tech. Univ. of Denmark, Build. 326 \\ DK-2800 Kgs. Lyngby, Denmark \\ hhn@elektro.dtu.dk
}

\author{
Niels Kjølstad Poulsen \\ Informatics and Math. Modelling \\ Tech. Univ. of Denmark, Build. 321 \\ DK-2800 Kgs. Lyngby, Denmark \\ nkp@imm.dtu.dk
}

\begin{abstract}
A new set-up for fault tolerant control (FTC) for stable systems is presented in this paper. The new set-up is based on a simple implementation of the Youla-Jabr-BongiornoKucera (YJBK) parameterization. This implementation of the YJBK parameterization will allow a direct and simple reconfiguration of the feedback controller. Another central part of fault tolerant control is fault diagnosis. The controller implementation can be applied directly in connection with both passive diagnosis (PFD) as well as with active fault diagnosis (AFD). The presented FTC set-up is investigated with respect to sensor reconfiguration. Actuator reconfiguration can be dealt with in a similar way.
\end{abstract}

\section{INTRODUCTION}

Fault tolerant control (FTC) has become an important area in the last years due to the increasing system complexity. It is important to be able to handle faults in controlled systems - and in a systematic way such that major accidents can be avoided. One of the drawbacks in feedback control is that the effects from a fault, somewhere in the closed loop system, might not be removed from the loop. Furthermore, in many cases, the effect will be amplified through the loop with a major reduction of the performance of the system as the results. In other cases, the closed loop systems will be unstable when faults occur in the loop.

In general, a fault tolerant controller is made up of mainly two parts, a diagnosis part and a controller reconfiguration part. A number of different concepts for FTC has been considered, see e.g. [4], [14], [21].

The focus in this paper is on the application of the YJBK parameterization in connection with FTC. An FTC architecture based on the original implementation of the YJBK parameterization was presented in [14]. One of the advantages in this architecture is that the controller is reconfigured through the YJBK transfer function. In connection with the analysis and design of FTC, this will allow us to use the results from the YJBK and the dual YJBK parameterization. Another important issue with the presented FTC architecture in [14] is the nominal feedback controller is not changed in connection with a reconfiguration. The controller is modified by an additional feedback loop designed with respect to the detected and diagnosed fault.
One of the drawbacks by using the standard implementation of the YJBK parameterization ([1], [2], [5], [19], [20]) as the basis for an FTC architecture is the complexity in the controller switching. A switch from a controller of the same order as the system will in general require a YJBK transfer function of three times the order of the nominal controller, [16]. A new implementation of the YJBK parameterization described in [12] will be the basic for a new FTC architecture. The new implementation of the YJBK parameterization has a more simple structure than the original/standard implementation. This will allow a more simple way to switch between different controllers through the YJBK transfer function. This new controller implementation exists in two versions as in the original implementation, [5]. Both versions of the new implementations have a structure that includes a residual vector. These residual vectors are used for an internal feedback in the controllers. Further, these residual vectors are also used directly in connection with the fault diagnosis in the FTC architecture. In the original implementation of the YJBK parameterization, only one of the versions include a residual vector can be applied in connection with fault diagnosis. It will therefore only be possible to get one FTC architecture based on the original implementation of the YJBK parameterization.

In this paper, the new FTC architecture will be based on the dual version of the implementation of the YJBK parameterization. It will be shown how it is possible to obtain both passive and active fault diagnosis and also controller reconfiguration based on the same set of input and output vectors.

Another relevant aspect in relation with FTC is the possibility to change sensors and actuators in connection with controller reconfiguration. When a fault has occurred in the system, it will in many cases be relevant to let the reconfigured controller use another set of actuators and sensors than the nominal controller. It is then possible to use more reliable actuators and sensors in the faulty case than in the fault free case. The case of changing sensors in connection with controller reconfiguration has been considered in the last part of the paper. The derived results can be extended to the general case where also actuator changes. 
The rest of this paper is organized as follows. Some preliminary results are given in Section II. Implementation of controllers are considered in Section III followed by fault diagnosis in Section IV. The FTC implementation is described in Section V. Change of sensors in connection with controller reconfiguration is considered in Section VI. The paper is closed by a Conclusion in Section VII.

\section{PREliminary Results}

\section{A. System set-up}

The system set-up is shortly introduced in the following. Let a general continuous-time stable MIMO system be given by:

$$
\Sigma_{P}:\left\{\begin{array}{l}
e=G_{e d}(\theta) d+G_{e u}(\theta) u \\
y=G_{y d}(\theta) d+G_{y u}(\theta) u
\end{array}\right.
$$

where $d \in \mathcal{R}^{r}$ is a disturbance signal vector, $u \in \mathcal{R}^{m}$ the control input signal vector, $e \in R^{q}$ is the external output signal vector to be controlled and $y \in \mathcal{R}^{p}$ is the measurement vector. The system description in (1) may depend on a number $(k)$ of parameters. Let $\theta_{i}, i=1, \ldots k$ denote the parametric deviations away from the nominal values, ie. $\theta_{i}=0, i=1, \ldots k$ in the nominal case. Let us for short arrange the deviations in a vector:

$$
\theta=\left(\theta_{1}, \cdots, \theta_{i}, \cdots, \theta_{k}\right)^{T}
$$

Furthermore, let

$$
\vartheta_{i}=\left(0, \cdots, \theta_{i}, \cdots, 0\right)^{T}
$$

which represent the situation with a fault or change in precisely one parameter. In many cases it will be possible to give an explicit expression of the connection between the system and the parametric faults (see [9], [10]).

Let the system be controlled by a stabilizing feedback controller given by:

$$
\Sigma_{C}:\{u=K y
$$

\section{B. Coprime factorization}

Let a coprime factorization of the system $G_{y u}$ from (1) and the stabilizing controller $K$ from (2) be given by:

$$
\begin{aligned}
G_{y u} & =N_{0} M_{0}^{-1}=\tilde{M}_{0}^{-1} \tilde{N}_{0}, & N_{0}, M_{0}, \tilde{N}_{0}, \tilde{M}_{0} \in \mathcal{R} \mathcal{H}_{\infty} \\
K & =U V^{-1}=\tilde{V}^{-1} \tilde{U}, & U, V, \tilde{U}, \tilde{V} \in \mathcal{R} \mathcal{H}_{\infty}
\end{aligned}
$$

The stability condition gives that:

$$
\left(\tilde{V} M_{0}-\tilde{U} N_{0}\right)^{-1}=Z \in \mathcal{R} \mathcal{H}_{\infty}
$$

or

$$
\left(\tilde{V} M_{0} Z-\tilde{U} N_{0} Z\right)=(\tilde{V} M-\tilde{U} N)=I
$$

where the related right coprime factorization of $G_{y u}$ is given by:

$$
G_{y u}=\left(N_{0} Z\right)\left(M_{0} Z\right)^{-1}=N M^{-1}, N, M, Z \in \mathcal{R} \mathcal{H}_{\infty}
$$

Equivalent for the left coprime factorization of $G_{y u}$ is given by:

$$
G_{y u}=\left(\tilde{Z} \tilde{M}_{0}\right)^{-1}\left(\tilde{Z} \tilde{N}_{0}\right)=\tilde{M}^{-1}(\tilde{N}), \tilde{N}, \tilde{M}, \tilde{Z} \in \mathcal{R} \mathcal{H}_{\infty}
$$

where $\tilde{N}$ and $\tilde{M}$ will satisfy:

$$
\left(-\tilde{Z} \tilde{N}_{0} U+\tilde{Z} \tilde{M}_{0} V\right)=(-\tilde{N} U+\tilde{M} V)=I
$$

The eight matrices $N, M, \tilde{N}, \tilde{M}, U, V, \tilde{U}$ and $\tilde{V}$ from (3), (5) and (6) will satisfy the double Bezout equation given by, see [19]:

$$
\begin{aligned}
\left(\begin{array}{ll}
I & 0 \\
0 & I
\end{array}\right) & =\left(\begin{array}{cc}
\tilde{V} & -\tilde{U} \\
-\tilde{N} & \tilde{M}
\end{array}\right)\left(\begin{array}{cc}
M & U \\
N & V
\end{array}\right) \\
& =\left(\begin{array}{cc}
M & U \\
N & V
\end{array}\right)\left(\begin{array}{cc}
\tilde{V} & -\tilde{U} \\
-\tilde{N} & \tilde{M}
\end{array}\right)
\end{aligned}
$$

Further, $Z$ and $\tilde{Z}$ are given by:

$$
\begin{aligned}
Z & =M_{0}^{-1} M \in \mathcal{R} \mathcal{H}_{\infty} \\
\tilde{Z} & =\tilde{M} \tilde{M}_{0}^{-1} \in \mathcal{R} \mathcal{H}_{\infty}
\end{aligned}
$$

\section{The YJBK Parameterization}

With the previous mentioned coprime factorization of the system $G_{y u}$ and the controller $K$, we can give a parameterization of all controllers that stabilize the system in terms of a stable transfer function $Q$, i.e. all stabilizing controllers are given by using a right factored form [19]:

$$
K(Q)=(U+M Q)(V+N Q)^{-1}, Q \in \mathcal{R} \mathcal{H}_{\infty}
$$

or by using a left factored form:

$$
K(Q)=(\tilde{V}+Q \tilde{N})^{-1}(\tilde{U}+Q \tilde{M}), Q \in \mathcal{R} \mathcal{H}_{\infty}
$$

Using the Bezout equation, the controller given either by (10) or by (11) can be realized as an LFT (linear fractional transformation) in the parameter $Q$ :

$$
K(Q)=\mathcal{F}_{l}\left(\left(\begin{array}{cc}
U V^{-1} & \tilde{V}^{-1} \\
V^{-1} & -V^{-1} N
\end{array}\right), Q\right)=\mathcal{F}_{l}\left(J_{K}, Q\right)
$$

where (12) is the same for both the right and the left form given in (10) and (11), respectively.

In the same way, it is possible to derive a parameterization in terms of a stable transfer function $S$ of all systems that are stabilized by one controller, i.e. the dual YJBK parameterization. Using the right form, the parameterization is given by [19]:

$$
G_{y u}(S)=(N+V S)(M+U S)^{-1}, S \in \mathcal{R} \mathcal{H}_{\infty}
$$

or by using a left factored form:

$$
G_{y u}(S)=(\tilde{M}+S \tilde{U})^{-1}(\tilde{N}+S \tilde{V}), S \in \mathcal{R} \mathcal{H}_{\infty}
$$


An LFT representation of (13) or (14) is given by:

$$
\begin{aligned}
G_{y u}(S) & =\mathcal{F}_{l}\left(\left(\begin{array}{cc}
N M^{-1} & \tilde{M}^{-1} \\
M^{-1} & -M^{-1} U
\end{array}\right), S\right) \\
& =\mathcal{F}_{l}\left(J_{G}, S\right)
\end{aligned}
$$

Further, $S$ is given by, [19]:

$$
S=\mathcal{F}_{u}\left(J_{K}, G_{y u}(S)\right)
$$

The dual YJBK transfer function $S$ will be a function of the system variations $\theta$, i.e. $S=S(\theta)$. The connection between $S$ and $\theta$ has been considered in details in [7]. Assuming that $\theta=0$ is the nominal value of $\theta$, then there will exist the following simple relation, [7]:

$$
S(\theta)=0, \text { for } \theta=0
$$

This relation will be applied in the following in connection with fault diagnosis.

\section{Controller ImPlementation}

The implementation of the YJBK parameterization can be done as described in e.g. [8], [9], [14], [19] and the dual implementation in [5]. These implementations include an inversion of $\tilde{V}$ or $V$, respectively. Further, a switch from a nominal controller $K$ to another controller $K_{i}$ through a use of the YJBK transfer function $Q$ result in a quite complex $Q$.

Some of these drawbacks with the normal implementation can be removed by using a new implementation of the YJBK parameterization. The implementation has been described in details in [12]. The implementation is shown in Fig. 1. A dual version is shown in Fig. 2. Both implementations are based on residual vectors, $\varepsilon$ or $\bar{\varepsilon}$ respectively, as described in next section. The two implementations are therefore called for residual based implementations.

$U(Q)$ and $\tilde{U}(Q)$ are (see (10) and (11)) given by:

$$
\begin{aligned}
& U(Q)=U+M Q \\
& \tilde{U}(Q)=\tilde{U}+Q \tilde{M}
\end{aligned}
$$

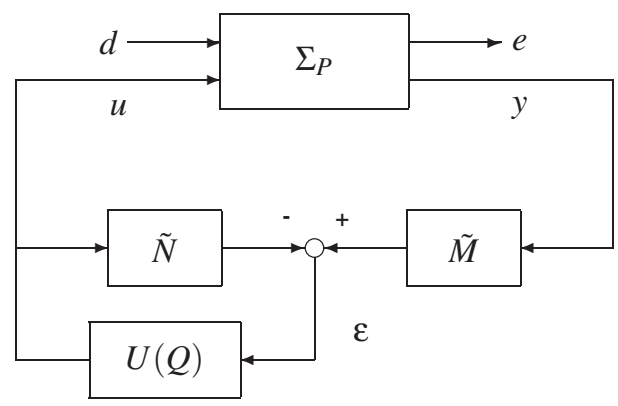

Fig. 1. A residual based implementation of the YJBK parameterization.

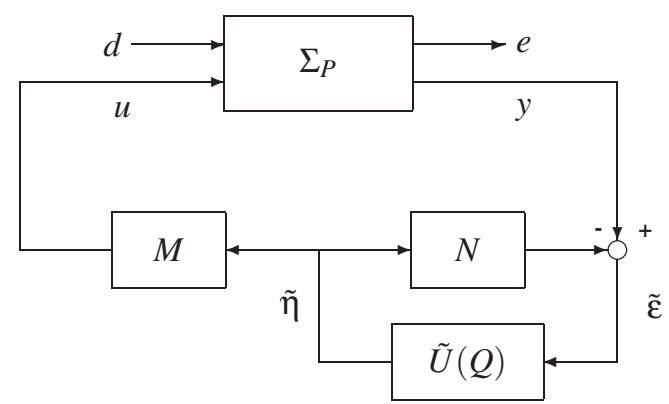

Fig. 2. A dual implementation of the YJBK parameterization shown in Fig. 1.

\section{A. Controller Switching}

Based on the YJBK parameterization, it is possible to switch from a nominal controller $K$ to another controller $K_{i}$ by a suitable selection of $Q$. Assume the existence of a coprime factorization of the system and the new controller

$$
\begin{aligned}
G_{y u} & =\left(N Z_{i}\right)\left(M Z_{i}\right)^{-1}=N_{i} M_{i}^{-1}, Z_{i}=M^{-1} M_{i} \\
& =\left(\tilde{Z}_{i} \tilde{M}\right)^{-1}\left(\tilde{Z}_{i} \tilde{N}\right)=\tilde{M}_{i}^{-1} \tilde{N}_{i}, \tilde{Z}_{i}=\tilde{M}_{i} \tilde{M}^{-1} \\
K_{i} & =U_{i} V_{i}^{-1}=\tilde{V}_{i}^{-1} \tilde{U}_{i}
\end{aligned}
$$

which satisfy the double Bezout equation given by:

$$
\begin{aligned}
\left(\begin{array}{ll}
I & 0 \\
0 & I
\end{array}\right) & =\left(\begin{array}{cc}
\tilde{V}_{i} & -\tilde{U}_{i} \\
-\tilde{N}_{i} & \tilde{M}_{i}
\end{array}\right)\left(\begin{array}{cc}
M_{i} & U_{i} \\
N_{i} & V_{i}
\end{array}\right) \\
& =\left(\begin{array}{cc}
M_{i} & U_{i} \\
N_{i} & V_{i}
\end{array}\right)\left(\begin{array}{cc}
\tilde{V}_{i} & -\tilde{U}_{i} \\
-\tilde{N}_{i} & \tilde{M}_{i}
\end{array}\right)
\end{aligned}
$$

Then a switching from $K$ to $K_{i}$ can be obtained by using $Q_{i}$ given by ([16]):

$$
Q_{i}=Z_{i}\left(\tilde{U}_{i} V-\tilde{V}_{i} U\right) \text { or } Q_{i}=\left(\tilde{V} U_{i}-\tilde{U} V_{i}\right) \tilde{Z}_{i}
$$

in (10) or in (11).

If the residual based implementation in Fig. 1 and Fig. 2 is applied, then it is impossible to reduce the complexity of the implementation of $Q_{i}$ significantly. Let $U(Q)$ in (18) be given by:

$$
U\left(Q_{i}\right)=U+\alpha M Q_{i}
$$

where $K$ is obtained for $\alpha=0$ and $K_{i}$ for $\alpha=1$. Including $Q_{i}$ in $U(Q)$ gives:

$$
U\left(Q_{i}(\alpha)\right)=(1-\alpha) U+\alpha U_{i} \tilde{Z}_{i}
$$

Equivalent, using $\tilde{U}(Q)$ in Fig. 2 gives

$$
\tilde{U}\left(Q_{i}(\alpha)\right)=(1-\alpha) \tilde{U}+\alpha Z_{i} \tilde{U}_{i}
$$

The equivalent $Q_{i}$ is given by

$$
Q_{i}=M^{-1}\left(U_{i} \tilde{Z}_{i}-U\right) \text { or } Q_{i}=\left(Z_{i} \tilde{U}_{i}-\tilde{U}\right) \tilde{M}^{-1}
$$

The closed loop transfer function from external input $d$ to external output $e$ is given by:

$$
\begin{aligned}
T_{e d} & =G_{e d}+G_{e u} U \tilde{M} G_{y d}+G_{e u} M Q \tilde{M} G_{y d} \\
& =G_{e d}+G_{e u} U(Q) \tilde{M} G_{y d}
\end{aligned}
$$


when the general feedback controller $K(Q)$ is applied.

Including $Q_{i}$ given by (23) in (24) gives directly:

$$
T_{e d}=G_{e d}+G_{e u} U_{i} \tilde{M}_{i} G_{y d}
$$

which show that the controller has been switched from $K$ to $K_{i}$.

\section{FAult Diagnosis}

In connection with fault diagnosis it is possible to apply both implementations of the feedback controller shown in Fig. 1 and in Fig. 2. In the following, the fault diagnosis will be considered only in connection with the controller implementation in Fig. 2.

Let the YJBK transfer function $Q$ be removed from the setup in Fig. 2. Further, including a reference $r$ input gives the set-up shown in Fig. 3. $\eta$ is an auxiliary input applied in connection with active fault diagnosis.

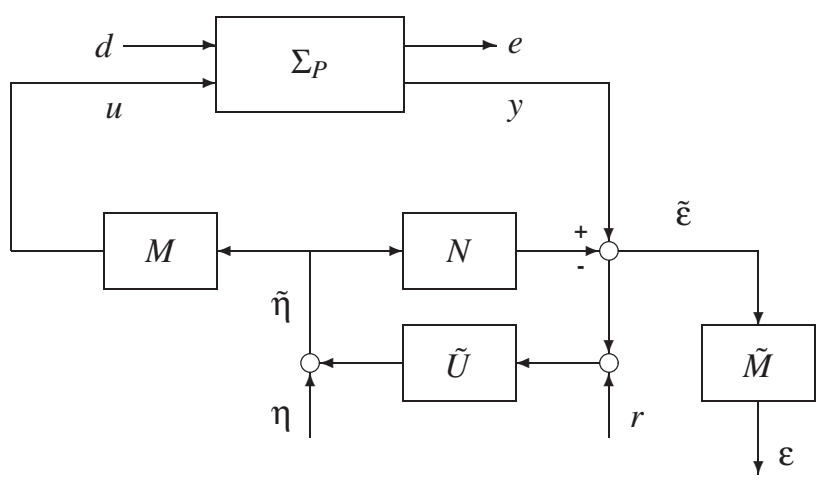

Fig. 3. A set-up for both passive and active fault diagnosis in closed-loop.

The transfer function from the inputs, $d, r$ and $\eta$ to the residual vector $\varepsilon$ can be derived in the following way:

$$
\begin{aligned}
\varepsilon & =\tilde{M}(I+N \tilde{U})^{-1}(y-N \tilde{U} r-N \eta) \\
& =V^{-1}(y-N \tilde{U} r-N \eta)
\end{aligned}
$$

Further, the output $y$ is given by:

$$
\begin{aligned}
y & =G_{y d}(\theta) d+G_{y u}(\theta) M\left(\eta+\tilde{U} r+\tilde{U} \tilde{M}^{-1} \bar{\varepsilon}\right) \\
& =G_{y d}(\theta) d+G_{y u}(\theta)(M \eta+M \tilde{U} r+U \bar{\varepsilon})
\end{aligned}
$$

This result in the following equation for $\varepsilon$ :

$$
\varepsilon=(\tilde{M}+S \tilde{U}) G_{y d}(\theta) d+S \tilde{U} r+S \eta
$$

where (13) and (14) has been applied in calculation of (26).

The residual vector $\varepsilon$ can be applied in connection with both passive and active fault diagnosis. In the passive approach $(\eta=0)$ the residual vector is given by:

$$
\varepsilon=\tilde{M} G_{y d}(0) d
$$

in the nominal case $(\theta=0)$. Changes in the system can be detected by using statistical tests directly on the residual vector to detect changes in the statistical properties such as mean and variance. Tests such as CUSUM and maximum likelihood methods can be applied, see e.g. [3], [6].

In the active approach, the auxiliary input $\eta$ is applied in order to enhance the precision of the diagnosis, which is based directly on the condition on $S$ given by (17). In the case of a fault, $S$ will be non-zero, and the detection is carried out a detection of the signature of $\eta$ in the residual. Fault isolation can be developed by an investigation of change of gain and phase properties through $S$. These changes depend directly of the parametric faults in the system. $S$ plays a central role in the AFD approach and has therefore also been named the fault signature matrix, [9], [11], [17], [18], wherein the AFD approach is described in details.

Note that the implementation in Fig. 3 based on the block diagram in Fig. 2 gives directly a residual vector that can be applied in connection with both passive and active fault diagnosis. This is not obtained in the original dual implementation of the YJBK parameterization. It has been pointed out in [21] that the missing naturally residual vector in the setup will make this implementation useless in connection with fault detection and fault tolerant control. This problem has been handled by using the new implementation in Fig. 2 or Fig. 3.

\section{FTC IMPLEMENTATION}

The implementation of a fault tolerant controller based on the controller implementation in Section III and the fault diagnosis part in Section IV is now described. The FTC implementation will be based on the dual implementation as shown in Fig. 2, because both controller switching and fault diagnosis can be derived from this architecture. The structure of a fault tolerant controller based on this controller implementation is shown in Fig. 4.

It is clear from the FTC set-up in Fig. 4 that both the reconfiguration and the diagnosis part are based on the same set of input and output vectors, i.e. $(\eta, \varepsilon)$. The set-up is shown in Fig. 4 is based on an AFD method. A passive diagnosis method can be included without changing the structure.

An important aspect of the shown set-up is that the structure of the diagnosis part is independent of the applied feedback controller. A reconfiguration of the feedback controller in terms of a non-zero $Q$ will not change the structure of the diagnosis part.

The design of the reconfiguration part $Q$ is not restricted to a specific method. A number of $Q$ 's can be designed offline or it is possible to using on-line optimization methods. It will depend on the specific application. Further, an advantage with the FTC set-up is that the nominal controller is not 


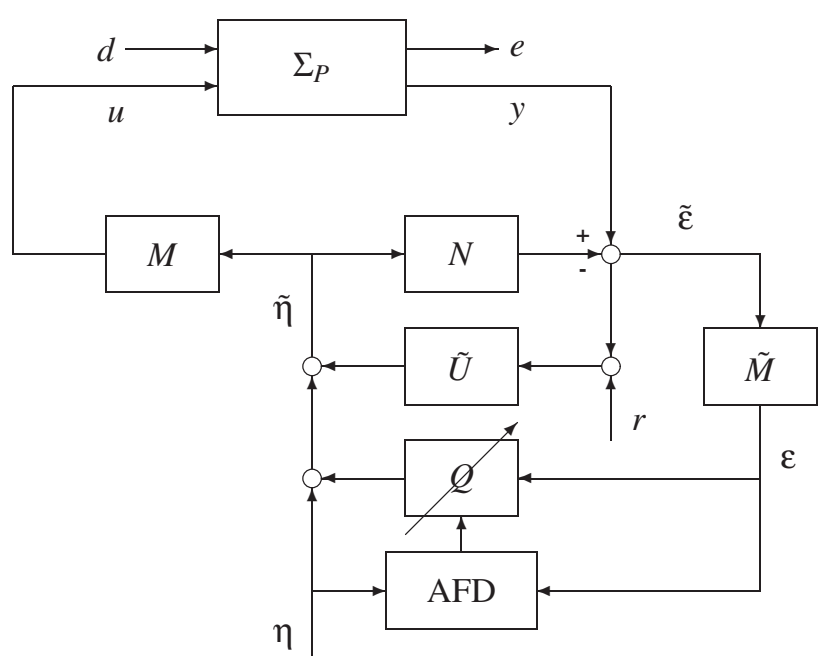

Fig. 4. A set-up for fault tolerant controllers based on the controller implementation in Fig. 2.

changes. It is then simple to switch back to the nominal controller. This can be done by using $Q=0$ in the controller.

As pointed out above, the structure of the diagnosis part does not need to be changed when the controller is reconfigured. To see this, let's consider the transfer function from the auxiliary input $\eta$ to the residual vector $\varepsilon$. For $Q=0$, the transfer function is given by:

$$
\varepsilon=S(\theta) \eta
$$

For $Q$ non-zero, the transfer function is given by:

$$
\varepsilon=S(\theta)(I-Q S(\theta))^{-1} \eta=S_{c l}(S(\theta), Q) \eta
$$

An important observation is that the condition for fault detection is independent of $Q$. I.e.

$$
\begin{aligned}
S(\theta)=0, & \text { for } \quad \theta=0 \\
S_{c l}(S(\theta), Q)=0, & \text { for } \quad \theta=0
\end{aligned}
$$

This important result has been discussed in more details in [9].

The condition for fault isolation will, in general, depend on the applied $Q$. The reason is that the output directions for the signature in $\varepsilon$ from $\eta$ depend strongly on the applied feedback controller. As a consequence of this, the test methods in the AFD block need to be modified when the controller is reconfigured. However, this aspect can also be used in a constructive way. In connection with fault isolation, after faults has been detected, it is possible to apply dedicated $Q$ 's to simplify the isolation of certain critical faults. This can be done by using $Q$ 's that will give a more significant and unique signature in the residual vector. It is of cause required that the applied $Q$ 's will not destabilize the system. This aspect will not be discussed further in this paper.

\section{Sensor Reconfiguration}

Reconfiguration of both sensors and actuators is a central element in connection with fault tolerant control. When fault has occurred in the system, one possibility might be to switch to a controller based on another sensor and actuator configuration. This includes also system faults and not only faults related to sensors or actuators. It is therefore relevant to consider an extension of the FTC architecture with extra sensors and actuators.

The general YJBK parameterization has been considered in connection with an extension of sensors and actuators in [8]. These results will be used in connection with the new FTC architecture presented in Section V. Only sensor reconfiguration will be described in the following. Actuator reconfiguration can be handled in an equivalent way.

\section{A. Sensor Faults}

First, let's consider the case with sensor faults. This will in general result in a reduction of available sensors. Let the general system description in (1) be described by:

$$
\Sigma_{P, \theta}:\left\{\begin{array}{l}
e=G_{e d} d+G_{e u} u \\
y=\left(I+\theta_{s}\right) G_{y d} d+\left(I+\theta_{s}\right) G_{y u} u
\end{array}\right.
$$

where $\theta_{s}$ represent sensor faults. A complete loss of a sensor is described by $\theta_{s, i}=-1$. The associated fault signature matrix $S\left(\theta_{s}\right)$ can be calculated explicit in this case. $S\left(\theta_{s}\right)$ is given by, [7]:

$$
S\left(\theta_{s}\right)=\tilde{M} \theta_{s}\left(I-N \tilde{U} \theta_{s}\right)^{-1} N
$$

The closed loop system is unstable if $S\left(\theta_{s}\right)$ is unstable. An unstable $S\left(\theta_{S}\right)$ require a reconfiguration of the feedback controller by in terms of stabilizing $Q$. $Q$ must satisfy

$$
\left(I-Q S\left(\theta_{S}\right)\right)^{-1} \in \mathcal{R} \mathcal{H}_{\infty}
$$

This result follows directly from the separation result described in [19] and later used in [13], [14], [15] in connection with FTC.

\section{B. Extension of Sensors}

Now, let's consider the general case where the system is extended with additional sensors. Let the parametric faults $\theta$ in the original system (1) be separated into sensor faults $\theta_{s}$ and other faults $\bar{\theta}$. I.e. $\theta=\left(\theta_{s}, \bar{\theta}\right)$. Further, let $\theta_{x}$ represent sensor faults in the additional sensors. Extending the general system in (1) with extra sensors will result in the following system:

$$
\Sigma_{P, \mathrm{ext}}:\left\{\begin{array}{c}
e=G_{e d}(\theta) d+G_{e u}(\theta) u \\
y_{\mathrm{ext}}=G_{y d, \mathrm{ext}}\left(\theta, \theta_{x}\right) d+G_{y u, \mathrm{ext}}\left(\theta, \theta_{x}\right) u
\end{array}\right.
$$


with

$$
\begin{aligned}
y_{\mathrm{ext}} & =\left(\begin{array}{c}
y \\
y_{s}
\end{array}\right) \\
G_{y d, \mathrm{ext}}\left(\theta, \theta_{x}\right) & =\left(\begin{array}{c}
G_{y d}(\theta) \\
G_{y d, s}\left(\bar{\theta}, \theta_{x}\right)
\end{array}\right) \\
& =\left(\begin{array}{c}
G_{y d}(\theta) \\
\left(I+\theta_{x}\right) G_{y d, s}(\bar{\theta})
\end{array}\right) \\
G_{y u, \mathrm{ext}}\left(\theta, \theta_{x}\right) & =\left(\begin{array}{c}
G_{y u}(\theta) \\
G_{y u, s}\left(\bar{\theta}, \theta_{x}\right)
\end{array}\right) \\
& =\left(\begin{array}{c}
G_{y u}(\theta) \\
\left(I+\theta_{x}\right) G_{y u, s}(\bar{\theta})
\end{array}\right)
\end{aligned}
$$

The index $s$ indicates the extra sensors. It has been shown in [8] that the coprime matrices have the following structure:

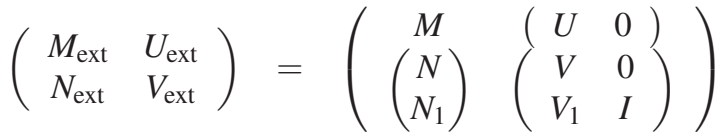

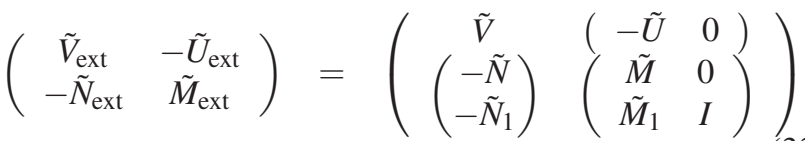

The above structure can always be obtained by using the state-space description for general controllers given in [19].

If the above coprime matrices are included in the dual controller implementation in Fig. 2, the extended implementation shown in Fig. 5 emerge. Here the YJBK transfer function for the extended system is given by:

$$
Q_{\mathrm{ext}}=\left(\begin{array}{ll}
Q & Q_{1}
\end{array}\right)
$$

where $Q_{1}$ is the transfer function due to the system extension.

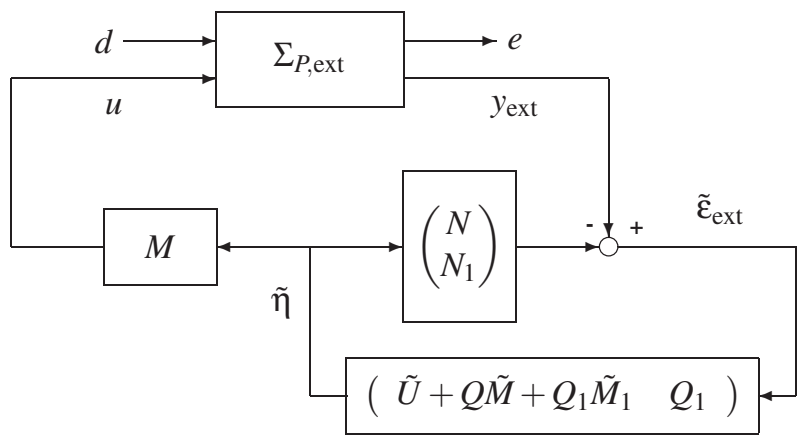

Fig. 5. A residual based implementation of the YJBK parameterization for a system with additional sensors.

\section{CONCLUSION}

A fault tolerant controller set-up based on a new implementation of the YJBK parameterized feedback controllers is described. This new controller implementation result in a simple method for controller reconfiguration. Further, it will also allow letting the FTC set-up be based on the YJBK parameterization in the right coprime form. Both fault diagnosis as well as controller reconfiguration are based on the same set of input and output vectors.

The problem of changing sensors in connection with controller reconfiguration is also considered. The case of changing actuators can be handled in the similar way. A change of sensors and actuators is relevant in connection with faults, where a controller for the faulty system might be based on other sensors and actuators. This is special relevant in connection with sensor and actuator faults.

\section{REFERENCES}

[1] B.D.O. Anderson. From Youla-Kucera to identification, adaptive and nonlinear control. Automatica, 34(12):1485-1506, 1998.

[2] P.J. Antsaklis and A.N. Michel. A Linear Systems primer. Birkhäuser, Boston, 2007.

[3] M. Basseville and I.V. Nikiforov. Detection of abrupt changes - theory and application. Prentice Hall, 1993.

[4] M. Blanke, M. Kinnart, J. Lunze, and M. Staroswiecki. Diagnosis and Fault-Tolerant Control. Springer, 2003.

[5] M.A. Dahleh and I.J. Diaz-Bobillo. Control of Uncertain systems. Prentice Hall, 1995.

[6] F. Gustafsson. Adaptive filtering and change detection. Wiley \& Sons, Chichester, England, 2000.

[7] H.H. Niemann. Dual Youla parameterization. IEE Proceedings Control Theory and Applications, 150(5):493-497, 2003.

[8] H.H. Niemann. Parameterization of extended systems. IEE Proceedings - Control Theory and Applications, 153(2):221-227, 2006.

[9] H.H. Niemann. A setup for active fault diagnosis. IEEE Transactions on Automatic Control, 51(9):1572-1578, 2006.

[10] H.H. Niemann and N.K. Poulsen. Active fault diagnosis in closed-loop systems. In Proceedings of the 16th IFAC World Congress, page 6p, Prague, Czech Republic, 2005.

[11] H.H. Niemann and N.K. Poulsen. Fault tolerant control for uncertain systems with parametric faults. In Preprints of 6th IFAC Symposium on Fault Detection Supervision ans Safety for Technical Processes, SAFEPROCESS'2006, pages 517-522, Beijing, China, 2006.

[12] H.H. Niemann and N.K. Poulsen. Controller architectures for switching. In Proceedings of the American Control Conference, pages 10981103, St. Louis, Missouri, USA, 2009.

[13] H.H. Niemann and J. Stoustrup. Reliable control using the primary and dual Youla parameterization. In Proceedings of the 41st IEEE Conference on Decision and Control, pages 4353-4358, Las Vegas, NV, USA, 2002.

[14] H.H. Niemann and J. Stoustrup. An architecture for fault tolerant controllers. International Journal of Control, 78(14):1091-1110, 2005.

[15] H.H. Niemann and J. Stoustrup. Passive fault tolerant control of a double inverted pendulum - A case study. Control Engineering Practice, pages 1047-1059, 2005.

[16] H.H. Niemann, J. Stoustrup, and R.B. Abrahamsen. Switching between multivariable controllers. Optimal Control - Application and Methods, pages 51-66, 2004.

[17] N.K. Poulsen and H. Niemann. Stochastic change detection based on an active fault diagnosis approach. In Proceeding of 46th IEEE Conference on Decision and Control, New Orleans, Louisiana, USA, December 2007.

[18] N.K. Poulsen and H.H. Niemann. Active fault diagnosis based on stochastic tests. International Journal of Applied mathematics and Computer Science, 18(4):487-496, 2008.

[19] T.T. Tay, I.M.Y. Mareels, and J.B. Moore. High performance control. Birkhäuser, 1997.

[20] K. Zhou, J.C. Doyle, and K. Glover. Robust and optimal control. Prentice Hall, 1995.

[21] K. Zhou and Z. Ren. A new controller architecture for high performance robust, and fault-tolerant control. IEEE Transactions on Automatic Control, 46(10):1613-1618, 2001. 